\section{Crisis y práctica filosófica en la educación}

Carlos Arturo Londoño Ramos

Doctor en Filosofía

Universidad Pedagógica y

Tecnológica de Colombia

https://orcid.org/0000-0002-6209-495x

londonocarlosarturo@hotmail.com

Jorge Aníbal Rojas Devia

Magíster en Educación

Universidad Pedagógica y

Tecnológica de Colombia

https://orcid.org/0000-0001-9731-3465

jorgeanibalrojas@hotmail.com
Artículo de investigación

Recepción: 14 de agosto de 2019 Aprobación: 05 de diciembre de 2019 https://doi.org/10.19053/22160159.v11.n25.2020.9910

\section{Resumen}

La enseñanza de la filosofía y el filosofar juegan un papel fundamental en la formación de seres humanos capaces de pensar racionalmente, comprender, argumentar, evaluar, reflexionar, cuestionar, criticar y proponer nuevas alternativas a la comprensión del saber y de los valores que se viven, desde los horizontes de las preguntas por los fundamentos y en la organización de las perspectivas. Los resultados de una educación filosófica adecuada tienen que ver con la formación de sujetos autónomos, éticos, capaces de vivir en democracia y autoconscientes de los criterios del saber. En este enfoque se circunscriben las competencias y la práctica filosófica que conforman un heterogéneo grupo de capacidades, vivencias y valores en el que la filosofía puede ser practicada en lugares, situaciones y contextos diferentes. Un enfoque más práctico de la filosofía hace que los estudiantes vean más aplicable esta disciplina, aumenta el gusto por su estudio 
y les permite el diálogo y el aprendizaje filosófico de las demás disciplinas. El objetivo de este trabajo reflexivo es comprender ese aprendizaje filosófico y su contribución a la enseñanza del filosofar.

Palabras clave: filosofía de la educación, enseñanza de la filosofía, práctica pedagógica, ética, humanidades

\section{Crisis and philosophical practice in education}

\section{Abstract}

The teaching of philosophy and philosophizing play a fundamental role in the training of human beings capable of thinking rationally, understanding, arguing, evaluating, reflecting, questioning, criticizing and proposing new alternatives to the understanding of knowledge and lived values, from the horizons of questions for foundations and in the organization of perspectives. The results of an adequate philosophical education are related to the training of autonomous, ethical subjects capable of living in democracy and who are self-conscious of the criteria of knowledge. In this approach, competencies and philosophical practice are defined, forming a heterogeneous group of abilities, experiences and values in which philosophy can be practiced in different places, situations and contexts. A more practical approach to philosophy makes students see this discipline more applicable, increases their taste for study and allows for dialogue and philosophical learning in other disciplines. The aim of this reflective work is to understand this philosophical learning as well as its contribution to the teaching of philosophy.

Keywords: philosophy of education, teaching of philosophy, pedagogical practice, ethics, humanities

\section{Crise et pratique philosophique dans l'éducation}

\section{Résumé}

L'enseignement de la philosophie et philosopher jouent un rôle fondamental dans la formation d'êtres humains capables de penser rationnellement, de comprendre, d'argumenter, d'évaluer, de réfléchir, de questionner, de critiquer et de proposer de nouvelles alternatives 
à la compréhension de la connaissance et des valeurs vécues, à partir des horizons des questions par les fondations et dans l'organisation des perspectives. Les résultats d'une éducation philosophique adéquate sont liés à la formation de sujets autonomes, éthiques, capables de vivre en démocratie et auto-conscients des critères de la connaissance. Dans cette approche, les compétences et la pratique philosophique sont délimitées, formant un groupe hétérogène de capacités, d'expériences et de valeurs dans lequel la philosophie peut être pratiquée dans différents lieux, situations et contextes. Une approche plus pratique de la philosophie fait que les étudiants voient cette discipline plus applicable, en augmentant leur goût pour l'étude et en permettant le dialogue et l'apprentissage philosophique dans d'autres disciplines. Le but de ce travail de réflexion est de comprendre cet apprentissage philosophique et sa contribution à l'enseignement de la philosophie.

Mots-clés : philosophie de l'éducation, enseignement de la philosophie, pratique pédagogique, éthique, sciences humaines

\section{Crise e prática filosófica na educação}

\section{Resumo}

O ensino da filosofia e filosofar desempenham um papel fundamental na formação de seres humanos capazes de pensar racionalmente, compreender, argumentar, avaliar, refletir, questionar, criticar e propor novas alternativas à compreensão do saber e dos valores que são vividos, desde os horizontes das perguntas por os fundamentos e a organização das perspectivas. Os resultados de uma educação filosófica adequada têm a ver com a formação de sujeitos autônomos, éticos, capazes de viver em democracia e autoconscientes dos critérios do saber. Neste enfoque circunscrevemse as competências e a prática filosófica que compõe um grupo heterogêneo de capacidades, experiências e valores em que a filosofia pode ser praticada em lugares, situações e contextos diferentes. Um enfoque mais prático da filosofia faz que os estudantes vejam mais aplicável esta disciplina, aumenta o gosto pelo seu estudo e permitelhes o diálogo e a aprendizagem filosófico das demais disciplinas. O objetivo deste trabalho reflexivo é entender essa aprendizagem filosófico e sua contribuição para o ensino de filosofar.

Palavras-chave: filosofia da educação, ensino da filosofia, prática pedagógica, ética, humanidades 


\section{Introducción}

El papel que juega la educación es fundamental en el nivel de desarrollo de una sociedad. Con el surgimiento de la era industrial, la educación se tornó utilitaria, es decir, una estrategia de formación necesaria para conseguir seres humanos profesionalizados en áreas específicas que demanda el mercado. A pesar de la formulación de modelos de enseñanza más abiertos, centrados en el estudiante y enfocados en la relación, entre acción, entendimiento einterés, como los de la pedagogía activa - en la tradición de Rousseau, Pestalozzi, Decroly, Dewey, Fröebel y Piaget, entre otros- la sociedad actual, en gran parte, mantiene los modelos magistrocéntricos en los que el estudiante es considerado como un ser vacío que debe ser llenado con información.

Así, las humanidades se han impartido, desde estos enfoques académicos y en algunos casos, tienden a desaparecer para dar cabida a asignaturas más útiles y de este modo se condena la democracia al fracaso (Nussbaum, 2013) y a la incomprensión de los saberes reducidos a formalismos o simplemente mecánicos.

Para comprender la función vital de la filosofía, como horizonte del pensamiento analítico, crítico y formativo, se requiere dar un rodeo por la ética greco-romana que promovía la renovación de la vida por medio de la filosofía. No obstante, como se pertenece a otra cultura, no es posible volver a otras épocas. Sin embargo, uno se puede inspirar en esa tradición filosófica por medio del método de la hermenéutica crítica, que consiste en la exploración y aclaración de textos para adecuarlos a la época moderna, como alternativa de libre opción para vivir de modo más cualificado, adquirir valores personales y aprender a saber ser y cuidar de sí (Mársico, 2017). No obstante, mediante este punto de vista se tiene que hacer frente a otros discursos dominantes y luchar en contra de perspectivas que pretenden limitar la educación a un saber simplemente profesionalizante.

Hoy más que nunca la filosofía debe reclamar un papel fundamental, en un mundo donde existe gran acceso a la información, pero donde pocos piensan, cuestionan y reflexionan sobre sí mismos y sobre las condiciones socioculturales; un país con bajos índices de lectura con un pobre nivel intelectual, y en el que sus ciudadanos acceden a medios de comunicación para 
el entretenimiento, para escapar de la rutina y no propiamente, para el conocimiento. En una sociedad, con una amplia historia de violencia, desigualdad, discriminación, corrupción, exclusión y superstición, se requiere de una formación en la cual se puedan cuestionar los saberes, conocer sus principios básicos, sus criterios, sus aplicaciones ejemplares, sus organizaciones y las diversas perspectivas existentes. Además, se necesita de un especial énfasis en una educación encaminada a fortalecer la democracia, las competencias valorativas de la personalidad y del entendimiento, así como una apropiación más comprensiva de las ciencias y disciplinas del saber (Acevedo \& Prada, 2017).

La desaparición de la filosofía en las aulas es un fenómeno ya reconocido en el mundo (Bain, 2006; Nussbaum, 2013; Unesco, 2011). Lo mismo sucede en Colombia, donde se reconoce que los saberes escolares de filosofía son "resúmenes diluidos y falsificados de los saberes de referencia de las universidades" (Serrano, como se citó en Redacción El Heraldo, agosto 14, 2016). Esta pedagogía tradicional aleja la formación de competencias críticas, dialógicas y creativas que se ha promovido desde el Ministerio de Educación Nacional (2010), en las Orientaciones pedagógicas para la filosofía en la educación media.

La ausencia de estas competencias transversales en la escuela genera serios problemas de desempeño en la universidad (Pulido, 2018), donde los estudiantes presentan grandes dificultades de comprensión del lenguaje para definir, clasificar, argumentar, generalizar y formular hipótesis o tesis. Tampoco han desarrollado la autonomía que les permita pensar por sí mismos y resolver los dilemas éticos a los que se enfrentarán en la vida (Zubiría, 2018). Sin embargo, a nivel mundial existen controversias sobre la presencia de la filosofía en la escuela:

En muchos casos, las iniciativas que han puesto énfasis en las materias aplicadas, técnicas o económicas tienen como consecuencia una reducción de la enseñanza de la filosofía en las escuelas y, en algunos casos, su supresión. En otros casos, resistencias de índole cultural o política han impedido una presencia más fuerte de la asignatura. (Unesco, 2011, p. 51)

Acerca de la idoneidad de la filosofía dentro de la educación, existe un debate, especialmente a nivel secundario. La controversia 
radica en la priorización de otras materias útiles para el desempeño laboral y la lucha dialéctica que se presenta con asignaturas relacionadas como religión y la educación moral (Unesco, 2011). En la mayoría de los casos, los estudiantes son adoctrinados en creencias, frente a las cuales no tienen capacidades de cuestionamiento; en la escuela el escepticismo, por lo general, no existe, pero tampoco las autoridades lo desean, no obstante, sin cierto grado de escepticismo no puede existir la pregunta, y en consecuencia, la creatividad intelectual.

La educación moderna necesita del pensamiento crítico (Perea, 2017). En las épocas del Estado confesional colombiano, la filosofía ocupaba más espacio y tenía mayor prestancia, pero, en lo fundamental, en la escuela secundaria la filosofía era la esclava de la teología en las creencias, las ciencias, la moral y la política (Londoño, 1985).

Paralelamente, en las últimas épocas, ya en un Estado laico, y en el proceso de postconflicto, se ha visto cómo las sociedades reclaman modelos de pensamiento que den alternativas de vida, ante la debilidad de esquemas religiosos, políticos y morales. Se requiere una cultura que reconozca la importancia de la búsqueda de sí mismo, del desarrollo personal y una mayor conciencia de la preponderancia de la apropiación critica de los saberes, del diálogo, de la argumentación, de la capacidad de abstracción, de razonamiento y de evaluación, es decir, del análisis, por encima de la transmisión de conocimientos (Unesco, 2011). A pesar de que, comparativamente, aún son pocos los que reclaman este tipo de modelos de pensamiento, en el mundo, se ha popularizado la práctica filosófica como alternativa para la vida; para pensar, reflexionar y hacerlo sobre el cuidado de sí.

\section{La crisis en la enseñanza de la filosofía}

Una de las causas fundamentales, por las que se debate la enseñanza filosófica en el sistema educativo, y que ocasiona su latente desaparición del currículo escolar, es la concepción mundial, en todos los niveles escolares, de las artes y las humanidades como ornamentos inútiles por quienes definen las políticas estatales, dando prioridad a todas las materias que sirven para ser competitivas en el mercado global (Nussbaum, 2013). Esta 
tendencia de pensamiento se ha extendido a la mentalidad de los padres de familia y los estudiantes. Así mismo, las pruebas de Estado ponen énfasis en las ciencias duras, es decir, aquellas materias útiles y prácticas para generar renta en los países; en consecuencia, el aspecto humanístico ha ido desapareciendo (Nussbaum, 2013). La imaginación, la creatividad y la rigurosidad en el pensamiento crítico han ido perdiendo terreno en la educación. Por lo tanto, la formación de estudiantes deshumanizados es un obstáculo para el logro de una sociedad libre y democrática, así como lo es para la creatividad científica y cultural.

En Sin fines de lucro: Por qué la democracia necesita de las humanidades, Nussbaum (2013) argumenta que el logro de este tipo de sociedad requiere de una lucha que debe librarse dentro de cada individuo para fortalecer facultades de pensamiento, actitudes, valores e imaginación, con el ánimo de reconocer su propia humanidad y la de los demás.

En gran parte, la formación filosófica escolar está anclada en una perspectiva histórica de repetición y de transmisión de contenidos, mientras que los métodos de enseñanza y evaluación se han fundamentado en el verbalismo. Esto ha implicado el desarrollo de clases que hoy siguen el patrón mencionado por Canfux (1996), en el que el profesor narra, expone información y exige al alumno memorización y la disertación se realiza, en torno a contenidos como segmentos de la realidad, aislados de su totalidad y ajenos a la experiencia existencial y a los saberes de los estudiantes. El resultado de este tipo de formación filosófica es el de sujetos que logran, en el mejor de los casos, hacer paráfrasis de los autores clásicos (León, 2006).

En un reciente congreso de filosofía realizado por la Universidad del Norte surgieron algunas inquietudes acerca de la enseñanza de la filosofía en Colombia. Por ejemplo, se vislumbran las falencias pedagógicas de los profesores de filosofía quienes están atados a su concepción limitada de la misma, como una de las causas del tipo de formación filosófica descrita anteriormente: "Muchos de los estudiantes universitarios salen de estudiar filosofía y van a los colegios a enseñar lo mismo, pero poquito, eso es un desastre porque no se estimula ese pensamiento crítico" (Díaz, como se citó en Redacción El Heraldo, 14 de agosto, 2016). El problema del 
sistema educativo es que está dedicado al aprendizaje de contenido y no al desarrollo del pensamiento, como ha sido el planteamiento de Piaget (1987). Esto también sucede con la enseñanza de la filosofía, que está enfocada para enseñar conceptos, grandes filósofos e historia y poco se trabaja en el ejercicio de filosofar.

Una consecuencia del debate, sobre la pertinencia de la enseñanza de filosofía, ha sido el mencionado cuestionamiento sobre su continuidad en el currículo escolar, esto es evidente con su desaparición de las pruebas de Estado: "Uno de los signos es que desde hace dos años ya no hay en las Pruebas Saber de once un apartado sobre filosofía, solo se evalúa indirectamente con las competencias de lectura crítica y el segmento de competencia ciudadana" (Tovar, como se citó en Redacción El Heraldo, 14 de agosto, 2016). Sin embargo, frente a la ausencia de contenidos filosóficos, la lectura crítica es dudosa porque no hay propiamente problemas y conceptos sobre los cuales poder ejercer la comprensión crítica.

Se observa entonces, a nivel mundial, una tendencia a la desaparición de la filosofía enmarcada en dificultades didácticas para hacerla más aprehensible y práctica. Tozzi (2007) adjudica parte de la responsabilidad de esta situación; al discurso antipedagógico de los representantes mayoritarios de la filosofía y a la, formación excesivamente académica de docentes, con una baja formación pedagógica en su profesión y que se oponen a reflexionar sobre una didáctica que permita la enseñanza filosófica de masas; también está la opinión de que la filosofía es una disciplina autosuficiente considerada como sabia, junto con la petulancia hacia otras disciplinas consideradas como menos sabias, instrumentales o plebeyas.

Por el contrario, en la tradición de la epistemología piagetiana existe cierto paralelismo entre el desarrollo filosófico, las ciencias y otras disciplinas (Piaget, 1987). En Colombia, la filosofía se imparte de acuerdo con la Ley 115 de 1994, en los grados 10 y 11, además del ámbito universitario; en algunos casos, las instituciones educativas impulsan y promueven el estudio de las humanidades y la filosofía, pero en su mayoría, la educación es básicamente tecnocrática. Desde las Orientaciones Pedagógicas para la Filosofía en la Educación Media del Ministerio de Educación Nacional (MEN, 
2010), se plantea la filosofía vinculada al aprendizaje de otros saberes y competencias como las comunicativas y ciudadanas.

Las orientaciones pedagógicas para la enseñanza de la filosofía plantean tres competencias: competencia crítica, como la capacidad para poner en entredicho las creencias y opiniones para llegar a la transparencia de los fenómenos, tal y como se dan en el mundo; competencia dialógica fundamentada en una antigua práctica del diálogo filosófico en el que "se reconocen y se critican mutuamente las razones, las pretensiones de verdad, de validez y de autenticidad, posibilitando así el desempeño de la argumentación profunda y el pluralismo necesario para vivir la democracia" y competencia creativa, en la que se espera que la reflexión filosófica estimule al estudiante en la emancipación del espíritu, la creación de formas alternativas de experimentar el mundo, entenderlo, representarlo y actuar en el mundo (MEN, 2010).

En contraste, en la época actual de capitalismo tardío, el ser humano se encuentra en una encrucijada respecto a su pensamiento y visión de vida, por lo tanto, ha retomado o se ha interesado más en filosofar (Unesco, 2011). Se ha llegado a dicha encrucijada conflictiva debido a diversos aspectos, entre otros: 1) El fracaso, la debilidad o desaparición de grandes esquemas ideológicos, políticos, morales y religiosos tradicionales. 2) La transformación de las modalidades socio-económicas tradicionales que obliga a pensar nuevos fundamentos ético-antropológicos y nuevos valores. 3) La banalización de la cultura semi-sicológica de auto-ayuda y esoterismo, que propugna la búsqueda de uno mismo, sin sustentos racionales y que desemboca, de manera natural, en la multiplicación de prácticas de pseudo-desarrollo personal, sin fundamentos propiamente argumentativos. 4) La forma del aprendizaje y de la investigación científica empobrecidos, pues privilegia los datos, la mecanización de la información y de la semi-formalización, por encima de la capacidad explicativa, de su relevancia y comprensión. 5) La inquietud por cambiar la valoración del pensamiento, a nivel educativo, es decir, la tendencia a privilegiar menos la trasmisión, para favorecer en cambio y sobre todo, la labor de la apropiación de los saberes, el diálogo, la comprensión, el análisis, entre otros. 6) El acceso a gran cantidad de información y desinformación de los medios de comunicación. 7) La creciente individualidad y la decadencia de los grandes modelos socioculturales que han 
promovido, en cada individuo, la necesidad de generar su propio conjunto de pensamientos, valores, razones de ser y finalidades que dan sentido a su existencia particular.

En este sentido, por fuera del entorno académico, existe una creciente necesidad de marcos, estrategias o esquemas que permitan pensar la vida, los saberes fecundos y la existencia de los individuos. Para Habermas (1989), en la Teoría de la acción comunicativa, a pesar del gran proceso de desertificación del espíritu, por efecto de la predominancia de la razón instrumental del poder y del dinero, se requiere ampliar la acción comunicativa argumentativa, en todos los ámbitos del saber; la verdad es un acuerdo entre nosotros, según la sustentación de las afirmaciones y refutaciones, las normas éticas con contratos sociales pactados, con pretensiones de consenso, y los valores son autoexpresiones de subjetividad, en contextos sociales y propuestos argumentativamente.

\section{Filosofía como forma de vida}

La filosofía como forma de vida es un ejercicio espiritual que difiere de la filosofía entendida solo como una acción intelectual o un discurso teórico. La filosofía fue vista como una forma de vida por los filósofos griegos como Sócrates, Platón y el estoicismo; una visión que se pierde en la época tardía del helenismo. A través de la historia, la filosofía ha conservado su carácter vital, sin embargo, la intensidad de este punto de vista se ha visto atenuada, especialmente, en los comienzos del cristianismo cuando fue desplazada a un segundo plano para dar prioridad a la salvación del alma; luego en la escolástica medieval, con la pretensión de racionalizar la teología $\mathrm{y}$, por último, en el academicismo del siglo XIX, cuando la filosofía toma un carácter de especulación teórica, de comentario o de doctrina académica; épocas en las que, como expresa Thoreau, ha habido "profesores de filosofía, pero no filósofos" (Thoreau, como se citó en Hadot, 2006, p. 275).

Los antiguos griegos concebían que el filósofo debe conducir su vida filosóficamente, debe ser capaz de llevar una vida filosófica, en consecuencia, el filósofo práctico debe cuidarse a sí mismo, por medio de la filosofía y luego, si es su querer, adquirir la capacidad para ayudar a los demás (Brenifier \& Arnaiz, 2011). En la modernidad, dentro de esta línea de pensamiento se encuentran, 
entre otros, y en muy diversas formas, filósofos como Descartes, Espinoza, Pascal, Voltaire, Montaigne, Schopenhauer, Nietzsche, Sartre y Jaspers, el comunitarismo como filosofía, y Foucault.

De acuerdo con Hadot en Qué es la filosofía antigua (1998), en la antigüedad los ejercicios filosóficos se orientaban a la toma de conciencia, una clase de desdoblamiento o toma de perspectiva en la que el yo "se concentra en sí mismo, descubriendo que no es lo que creía ser, que no se confunde con los objetos a los cuales se había apegado" (Hadot, 1998, p. 209). La filosofía ha de ser utilizada como una herramienta de autoexamen y adquisición de conciencia sobre los aspectos de la vida. Para Hadot, en la obra Los ejercicios espirituales y la filosofía antigua (2006), la filosofía es una forma de vivir en la verdad, en el bien, en sabiduría, en ser mejor. Esto lo evidencia, a partir de la descripción de los filósofos griegos y romanos, especialmente a partir de Sócrates:

La filosofía consistía en un método de progresión espiritual que exigía, una completa conversión, una transformación radical de la forma, de ser. La filosofía constituía, pues, una forma de vida, y su tarea y práctica iba encaminada a alcanzar la sabiduría. [...] La sabiduría era una forma de vida que traía aparejada la serenidad de espíritu (ataraxia), la libertad interior (autarkeia) y la consciencia cósmica. En primer lugar, la filosofía se presentaba como una terapéutica destinada a curar la angustia. (Hadot, 2006, pp. 236-237)

Para Hadot (2009), la filosofía no es solamente un discurso, sino un ejercicio de sabiduría, un ejercicio espiritual, una elección de vida. La enseñanza de la filosofía como un discurso teórico ha formado en los estudiantes la impresión de que cada filósofo, en su momento, se afanó por inventar, de manera original, una construcción abstracta y sistemática y, a partir de estas construcciones teóricas, se derivan consecuencias para el comportamiento del hombre y la sociedad, sin negar la capacidad extraordinaria de los filósofos antiguos para desarrollar reflexión teórica de los problemas más sutiles de la vida.

Hadot (1998) indica que se ha entendido erróneamente la filosofía antigua y la elección por un modo de vida no se localiza al final del proceso de la actividad filosófica, sino en su origen "en una compleja interacción entre la reacción crítica a otras actitudes existenciales, la visión global de cierta manera de vivir y de ver el 
mundo, y la decisión voluntaria misma" (Hadot, 1998, p. 13). La elección de una opción existencial implica una visión del mundo, y la tarea del discurso filosófico es justificar y revelar racionalmente dicha opción existencial como su representación del mundo. Por lo tanto, el discurso filosófico se origina en una elección de vida y una opción existencial y no al revés; la elección de vida del filósofo determina su discurso (Hadot, 1998).

En otras palabras, la elección de cierto modo de vivir, una opción existencial, de un saber ser, exige del individuo un cambio total de vida, una conversión de todo el ser y el deseo de ser y vivir de cierto modo; dicho modo de vivir construye un discurso que justifica, motiva e influye en esta elección de vida (Hadot, 1998). De este argumento se deriva que "el discurso es filosófico únicamente, en la medida en que se transforma en modo de vida" (Hadot, 1998, p. 190). Modo de vida y discurso no son opuestos, pues no se trata de la contraposición entre práctica y teoría. De acuerdo con Hadot (1998), el discurso tiene un aspecto práctico, si produce un efecto en el auditor o lector y el modo de vida puede ser teorético o contemplativo.

A conclusiones similares llegaron otros autores. Foucault (1999) ve en la filosofía una forma de llegar a ser otro distinto al que se es; Nussbaum (2013) plantea que estudiar el mundo interior y su relación con las condiciones sociales es una tarea necesaria y suficiente para la filosofía política que aspire a ser práctica; y Nehamas (2006) contempla la filosofía como un mecanismo de transformación y perfeccionamiento del sujeto con fines estéticos, es decir, convertir su existencia en una obra de arte, en un logro literario y filosófico, más allá de las necesidades históricas, políticas o psicológicas.

Uno de los grandes problemas que presenta este planteamiento es el de la diferencia entre las sociedades del capitalismo tardío y la vida en la Ciudad Estado antigua, en la que este modo de vida generaba una valoración del ciudadano por sus virtudes cívicas honorables. En gran parte, esa aprobación social se ha perdido y todo su lenguaje fue connotado de modo religioso, en especial en América Latina. En la modernidad tardía se tienen otros valores personales y principios éticos constitucionales universalistas que favorecen la vida privada, pero también se ha generado un gran vacío por el agujero que ha 
dejado la decadencia de las religiones. Estas condiciones conducen al planteamiento de una nueva ética, una nueva moral sexual (Foucault, 1991), nuevas virtudes, mejores formas de alcanzar la calidad de vida y excelencias que se constituyen en méritos y valores personales que no pueden ser obligatorios ni universales, pero que podrían ser políticas educativas laicas tanto para los padres de familia como para las escuelas, los movimientos sociales y el Estado en la formación de las juventudes (Londoño, 2002).

Según las filosofías liberales, no es el papel del Estado el de entrometerse en la vida privada de los ciudadanos, por esa razón, la ética comunitarista que defiende las virtudes, a favor de nuevas formas de calidad de vida, debe ser compatible con el liberalismo, pues de lo contrario, culminaría en un totalitarismo. El cuidado de sí es una opción personal meritoria.

\section{Cuidado de sí mismo}

La filosofía como modo de vida está relacionada con el cuidado de sí mismo. Para Foucault, en las Tecnologías del yo (1990), la noción del cuidado de sí, originada en la filosofía socrática, es un concepto menor y descuidado de la historia de la filosofía debido a un largo proceso histórico que desplaza el foco de la existencia de la vida hacia el conocimiento, que él denomina momento cartesiano. Para Kohan (2009), el momento cartesiano practica un juego doble:

a) Valoriza el conocimiento de sí tomando como punto de partida del recorrido filosófico la evidencia, que solo se puede dar desde el propio sujeto que conoce en cuanto alma, [como cosa pensante] res cogitans y, b) desvaloriza el cuidado de sí, indicando que no hay ni puede haber otro acceso a la verdad que el conocimiento emanado de la res cogitans. (p. 49)

En este momento cartesiano es en el que dos asuntos que estaban juntos, toman rumbos distintos: cuidado-conocimiento, vida-verdad, espiritualidad-filosofía; el cuidado y la espiritualidad quedan por fuera del conocimiento y son ubicados del lado de la vida. Otra razón, atribuida por Foucault en La hermenéutica del sujeto (1994), es que la relegación por el cuidado de sí mismo en la filosofía occidental ha sido la integración de esta preocupación en la moral del no-egoísmo en el cristianismo. No obstante, la razón más profunda de este abandono ha sido el acento más marcado en 
el conocimiento de uno mismo, como la vía fundamental del acceso a la verdad (Foucault, 1994).

En este sentido, Foucault planteó el cuidado de sí mismo épiméleia - como el "conjunto de prácticas mediante las cuales un individuo establece cierta relación consigo mismo y en esta relación el individuo se constituye en sujeto de sus propias acciones" (1990, p. 189). El cuidado de sí consiste en ocuparse de la existencia, alma, cuerpo, acciones, pensamientos, deseos, de tal manera que una vez se logre, pueda cuidar a otros. Dentro de este concepto de cuidado de sí, Foucault (1994) distingue tres aspectos que pueden entenderse como las dimensiones del mismo:

- Actitud: un determinado modo de enfrentarse al mundo, de comportarse, es una actitud general en relación con uno mismo y con los otros.

- Atención: una determinada forma de atención y de mirada desplazada desde el exterior, desde el mundo, desde otros, hacia sí mismo. Se puede entender también como la vigilancia sobre lo que uno piensa y lo que acontece en el pensamiento.

- Actuación: una determinada forma de actuar para hacerse cargo de uno mismo, en la que se modifica, se purifica, se transforma o se transfigura.

\section{Ejercicios espirituales filosóficos}

La espiritualidad consiste en la indagación, práctica y experiencias que utiliza el sujeto para transformarse y acceder a la verdad. Dentro de esta espiritualidad, Foucault (1994) señala tres características:

1) La única forma de acceder a la verdad es la de transformarse a sí mismo en algo distinto, pues esta no le es concedida al sujeto de pleno derecho. El precio de la verdad es la conversión del sujeto.

2) La conversión se realiza, por medio del trabajo que el sujeto realiza sobre sí mismo para convertirse finalmente, en un sujeto capaz de lograr la verdad mediante un movimiento de ascesis.

3) La verdad es lo que ilumina al sujeto, es decir, el acceso a la verdad produce una retroalimentación de la verdad sobre el sujeto. 
Teniendo en cuenta estas tres características es comprensible que el conócete a ti mismo, popularizado en la filosofía occidental, no sea suficiente para acceder a la verdad si no está acompañado de una transformación del sujeto, y esta transformación tampoco puede darse si no existe un ejercicio, una práctica sobre sí mismo. En otras palabras, la verdad no llega por el solo acto de ser sujeto - por derecho propio- o por el único acto de conocimiento de sí mismo, debe acompañarse con una metamorfosis del sujeto. De aquí también se desprende un concepto ético de la verdad, la cual no es aquello que se encuentra por el acto de conocimiento o para completar ese acto de conocimiento; "la verdad es lo que ilumina al sujeto, lo que le proporciona la tranquilidad de espíritu" (Foucault, 1994, p. 39). La verdad es la que perfecciona al sujeto o lo transfigura. Para la espiritualidad, la verdad y el acceso a la verdad ejercen una influencia en el sujeto y produce cambios que lo hacen mejor.

Entonces, es posible observar que la referencia al ejercicio, la transformación y el cuidado de sí, implica que la filosofía es una práctica, una actividad, un trabajo sobre sí mismo. Esta concepción de la filosofía tiene consecuencias en su enseñanza y en las metodologías para hacer filosofía. Los ejercicios espirituales son diversos: como las prácticas que podrían ser discursivas, como el diálogo y la meditación, o intuitiva, como la contemplación. Lo común a todas ellas es que estaban destinadas a generar una transformación del sujeto (Hadot, 1998, p. 15).

Se observa entonces, que los ejercicios espirituales, por definición, implican prácticas en el orden físico, discursivo, espiritual o reflexivo que como condición necesaria tienen la modificación del sujeto que las practica. En la Antigüedad, los ejercicios espirituales eran tan diversos, según las escuelas filosóficas que se traten, sin embargo, detrás de esta aparente diversidad Hadot (2006), encuentra que existe una profunda unidad en los medios utilizados, como en la finalidad buscada: "Los medios utilizados abarcan técnicas retóricas y dialécticas persuasivas, intentos de control del leguaje interior, concentración mental. La finalidad que pretenden tales ejercicios y, en general, todas las escuelas filosóficas consisten en la realización y mejora de uno mismo" (Hadot, 2006, pp. 48-49).

Los ejercicios espirituales tienen en común el uso de prácticas de diálogo externo e interno y meditación para alejarse de las pasiones, 
preocupaciones, de la confusa inquietud, de la falta de poder sobre sí mismo, delos prejuicios humanos y delas conveniencias sociales. Hadot (2006), explica que, así como el ejercicio físico fortalece el cuerpo y da mejor apariencia, con los "ejercicios espirituales proporciona más vigor a su alma, modifica el paisaje interior, transforma su visión del mundo y, finalmente, su ser por entero" (p. 49). La premisa fundamental que existe en los ejercicios espirituales es que la felicidad, la plenitud y la perfección existen dentro del ser humano, solo hay que encontrarlas, al despejar todo aquello que sobra; tal como el que esculpe su propia estatua esla realización de uno mismo. Tallabor deesculpir se encuentra en el contenido conceptual de los ejercicios espirituales: tranquilidad de ánimo, fortaleza y sometimiento al destino - estoicos-; serenidad y desapego -epicúreos-; concentración mental y contemplación de las ideas - platónicos-.

Los ejercicios espirituales son clasificados por Hadot (2006) en cuatro funciones básicas:

Aprender a vivir: consiste en usar la filosofía para controlar las pasiones y recuperar la alegría por el simple hecho de existir y hay diversos ejercicios espirituales que permiten lograrlo: atención, meditación, rememoración de cuanto es beneficioso, lectura, escucha, estudio, examen en profundidad, dominio de uno mismo, cumplimiento de los deberes, indiferencia ante las cosas que no están en nuestra decisión, contemplación y el vivir el presente, entre otras.

Aprender a dialogar: la práctica de un ejercicio espiritual es dialógica solo si supone un auténtico ejercicio de presencia, tanto ante uno mismo, como ante otros (Hadot, 2006). Por ejemplo, el diálogo socrático es un ejercicio espiritual practicado en común que permite dirigir la atención sobre uno mismo, al examen de conciencia. De acuerdo con este autor, solo quien es capaz de un verdadero encuentro con el otro está en disposición de encontrarse auténticamente consigo mismo y viceversa.

Aprender a morir: el ejercicio de aprender a morir consiste en cambiar la perspectiva de lo que es inevitable; la muerte, para tomar conciencia y valorar el momento presente y mantener serenidad ante las desgracias. Esto es posible cuando el alma se eleva al plano del pensamiento: desde lo individual hasta la universalidad. Gracias a esta elevación se pueden comprender los asuntos humanos como insignificantes, incluso la muerte. 
Aprender a leer: teniendo en cuenta como precepto que en la Antigüedad, la práctica de ejercicios espirituales fundamentó las teorías filosóficas, estas últimas se pueden leer sin tener en cuenta esta perspectiva concreta que nos señala su verdadero significado (Hadot, 2006). Aprender a leer implica prestar la máxima atención a la actitud existencial que cimenta el edificio de estas teorías, pues estas surgen en el contexto en el que un maestro educa a sus discípulos intentando conducirles a la transformación y realización de sí mismos. Las obras antiguas están pensadas en el eventual interlocutor y en su transformación. Implícitamente plantean un diálogo.

\section{¿Qué es enseñar filosofía?}

La palabra educar tiene un origen etimológico dual, se atribuye al latín educere y educare. El primero, indica conducir fuera de o extraer de dentro hacia afuera, mientras que el segundo, significa criar o alimentar (Luengo, 2004). Esta etimología dual también indica que la filosofía es un método, un procedimiento: el proceso del filosofar simultáneamente es un aprendizaje, una problemática y una temática que hay que enseñar.

Las nociones filosóficas, que se han construido históricamente, no son piezas decorativas o de museo, cuya función no sea más que ser admiradas tras la vitrina de la historia de la filosofía; por el contrario, dichas nociones deben ser estudiadas para responder a estos problemas o abordajes que fueron creados y así poder usarlas como herramientas de trabajo para comprender la realidad (Espinel \& Pulido, 2017). Esto implica que la educación tradicional de filosofía ha venido utilizando las nociones y escuelas de pensamiento filosófico como una tarea memorística y de acumulación de conocimiento, más que una aplicación a la vida misma. Teniendo en cuenta los planteamientos de Hadot (2006), en donde la experiencia y modo de vida del filósofo construyen el discurso filosófico, el estudio de la historia de la filosofía, especialmente, de los textos antiguos, debe hacerse develando la actitud existencial que fundamenta el edificio de estas teorías, cómo dichos textos buscaban conducir a sus discípulos a la transformación y al cuidado de sí mismos y qué elementos van a poder contribuir al cuidado de sí de los estudiantes. 
El estudio de los textos filosóficos no es una tarea para acumular conocimiento, sino una tarea de complemento de la práctica filosófica. Cerletti (2008) retoma a Kant (1943), quien plantea que no se puede aprender filosofía, pues no la ha habido aún, es decir, no existen pensamientos terminados y duraderos dentro de esta disciplina; incluso si existiera, tampoco serían permanentes pues dichos pensamientos se caracterizan por ser históricos. De esta manera, Kant, en Sobre el saber filosófico (1943), plantea que el filósofo no es un imitador ni un farsante:

El verdadero filósofo tiene que hacer, pues, como pensador propio, un uso libre y personal de su razón, no servilmente imitador. Pero tampoco un uso dialéctico, esto es, tal que solo se proponga dar a los conocimientos una apariencia de verdad y sabiduría. (p. 46)

La visión integral de Obiols (2008) debe resaltarse, pues según él, la enseñanza de la filosofía debe incluir el estudio histórico de la misma, como punto de distinción del intento personal de filosofar. Por lo tanto, la filosofía y el filosofar son sustantivo y verbo indispensables e inescindibles para que el aprendizaje de contenidos o habilidades pueda considerarse filosófico. Así mismo, filosofar no consiste en preguntar y buscar respuestas sin un método o sin buscar el fundamento de los problemas.

Para Brenifier y Arnaiz (2011), la esencia del filosofar está en "ir más allá de nuestra opinión personal" (p. 39). De este modo, existen tres dimensiones del filosofar que intentan fomentar las capacidades de pensar por sí mismo, ser uno mismo y ser dentro de la colectividad:

- Intelectual, piensa por sí mismo: pregunta, objeta y argumenta; propone conceptos, tesis e hipótesis; estructura, articula, clarifica, analiza,distingue y sintetiza; comprende las ideas de los demás y las de sí mismo; reformula o modifica las ideas. Trabaja en la relación entre el ejemplo y la idea. Se inicia en la lógica: la definición, la relación entre los conceptos, la coherencia de las ideas, el desarrollo del juicio y del discurso. El intelectual utiliza y crea instrumentos conceptuales de evaluación en referencia: al error, la falsedad, la verdad, lo absurdo, la identidad, la diferencia, los contrarios y las contradicciones, etcétera; constata la comprensión y el sentido de una idea. 
- Existencial, ser uno mismo: consiste en singularizary universalizar el pensamiento; expresar y asumir la identidad personal, a través de las elecciones y juicios; ser consciente de uno mismo, de sus ideas y de su comportamiento; controla las reacciones emocionales; trabaja la propia forma de ser y el pensamiento; se interroga, descubre y reconoce el error y la incoherencia en uno mismo; ve, acepta, verbaliza y trabaja los propios límites; se distancia de su forma de ser, de sus ideas y de sí mismo.

- Social, pensar con los otros: escucha al otro, le proporciona su espacio, respeto y comprensión; se interesa por el pensamiento del otro: se descentra, por medio de la reformulación de las preguntas y del diálogo; se arriesga y se integra a un grupo: permite que los otros lo pongan a prueba; comprende, acepta, discute y aplica las reglas de funcionamiento; se responsabiliza: modifica el estatus del alumno frente al del maestro; piensa con los otros y, dado el caso, compite con ellos.

Las actividades planteadas dentro de cada una de estas dimensiones del filosofar, permiten ver que no es un simple ejercicio de debate de opiniones ni que enseñar filosofía es solamente trasmitir conocimientos de filosofía. Enseñar filosofía es enseñar a filosofar, es enseñar a preguntar sobre criterios del saber, horizontes, globalidades, perspectivas y a buscar respuestas. El filosofar y el preguntar están fundamentados en una actitud filosófica, de sospecha, cuestionadora o crítica (Cerletti, 2008), lo cual implica una disposición de asombro, duda e incertidumbre acerca de las cosas. De acuerdo con Cerletti (2008), consisten en una mirada aguda y radical que no deja nada sin revisar, en problematizar las afirmaciones o poner en duda aquello que se presenta como obvio, natural o normal.

De acuerdo con Brenifier en Sobre el diálogo socrático (s. f.), la actitud filosófica es una disposición para que la actividad reflexiva pueda ejercerse. El autor presenta siete actitudes que el docente debe formar en sus estudiantes:

Sosegarse: se trata de calmar el pensamiento y el cuerpo, salir de la precipitación por hablar y equilibrarse, según el ritmo de trabajo que puede ser una lección, trabajo escrito o un diálogo. También, se debe actuar más intencionadamente, con mayor conciencia y de manera menos reactiva. 
Ignorancia adquirida: radica en introducir la incertidumbre, suspender el juicio, abandonar por un momento lo que se sabe y superar las respuestas únicas, buenas, absolutas o poderosas, para trabajar más en el proceso de reflexión común y la problematización.

Autenticidad: estriba en actuar sin buscar la aprobación de los demás, es pensar, decir lo que se piensa, arriesgarse a hacer hipótesis y ser responsable ello.

Empatía, simpatía: se basa en saber ponerse en el lugar de otro, comprender su punto de vista, ver desde otra perspectiva e identificarse con él. Consiste en estar dispuesto a entender la palabra extraña sin prejuicios, ni animosidad, pero con interés.

Confrontación: un compromiso con la crítica y el debate, ser capaz de confrontarse con el pensamiento de otro y el propio. Aunque esta actitud requiere el respeto por las ideas de otros, no significa tener una tolerancia floja, es decir, requiere un rigor en el que no se deben temer los unos a los otros.

Asombro: reconocer con sorpresa otros puntos de vista y el propio, permite que el pensamiento interrogue y capte verdades desconocidas.

Confianza: una actitud filosófica requiere confianza, en el otro, en uno mismo. Sin la confianza mutua cada uno permanecerá en la defensa de su punto de vista y en la negación para admitir errores.

El medio para profundizar filosóficamente son las preguntas, según Cerletti (2008), una pregunta filosófica se diferencia de los demás interrogantes por el tipo de respuesta que espera el que la formula. Se enraíza en la aspiración al saber, pero un saber sin supuestos. Por lo tanto, para una pregunta filosófica no es suficiente una primera respuesta, la cual queda interrumpida al volver a preguntar por los primeros supuestos, hasta llegar al corazón del concepto. A diferencia de la pregunta científica que se conforma con respuestas satisfactorias bajo un campo de estudio, una pregunta filosófica aspira a hacer explícitas las condiciones de producción y reproducción de los saberes y prácticas consagradas, razonadas y reflexivas. Estas se distinguen de las preguntas cerradas, retóricas o absurdas. 


\section{Conclusiones}

La filosofía como forma de vida es una manera de vivir en la interrogación por la verdad, el conocimiento, el bien, la justicia y la sabiduría. Igualmente, es un autoexamen en la adquisición de conciencia. Dentro de este contexto se encuentran las prácticas del cuidado de sí mismo, donde el sujeto se preocupa de sus acciones, de su existencia, de su entendimiento y de su cuerpo. Los ejercicios espirituales conducen a la transformación de sí mismo, como el precio que hay que pagar para acceder a la verdad; la práctica o el ejercicio implican cierta actividad física, discursiva, espiritual o reflexiva sobre sí mismo.

La praxis filosófica es la aspiración o deseo de saber, más allá del dominio de un saber determinado. Por lo tanto, filosofar no es un recorte de conocimiento que se va a trasmitir, sino una actividad en la que se aspira a alcanzar tanto los fundamentos como cierta orientación de totalidad del saber. La actividad filosófica y la enseñanza del filosofar no se pueden desligar de la producción filosófica ni de su enseñanza. La enseñanza del filosofar no es transmitir un recorte ocasional del conocimiento filosófico, en su lugar, consiste en generar la inquietud, formularse preguntas y buscar respuestas; en últimas, es ir más allá de la opinión personal, a través del auto-cuestionamiento y la comunicación con otros. De esta manera, el filosofar está ligado al hacer.

Las prácticas filosóficas son ejercicios en los que el sujeto se adentra en la búsqueda de la verdad y la sabiduría en la deliberación, a través del diálogo con otros. Dichas prácticas implican confrontar las teorías en la alteridad, en la comunicación: el ejercer el pensamiento con uno mismo, con el otro, con el mundo. En este sentido, filosofar consiste en preguntarse y buscar respuestas. En esta lógica, tanto el docente de filosofía como el de otras disciplinas, tiene ante sí diferentes formas de filosofar, las cuales, con la adecuada rigurosidad filosófica, en su implementación, pueden contribuir en la formación de seres humanos más críticos, interrogadores, evaluadores con criterios, autónomos, libres, racionalmente argumentativos y democráticos. Los sujetos que aprenden a filosofar y a llevar una vida filosófica estarán en la capacidad de incorporar las prácticas filosóficas al aprendizaje y a la discusión, en otros campos del conocimiento. 


\section{Referencias}

Acevedo, D., \& Prada, M, (2017). Pensar la vida: Crisis de las humanidades y praxis filosófica. Revista Colombiana de Educación, (72), 15-37. https://doi.org/10.17227/01203916.72rce15.37

Bain, K. (2006). Lo que hacen los mejores profesores universitarios. València: Universitat.

Brenifier, O. (s. f.). Práctica filosófica. Recuperado de https://www.ugr. es/ filosofia/materiales/textos/Brenifier/Brenifier_Competenciasfilosoficas.pdf

Brenifier, O., \& Arnaiz, G. (2011). Filosofar como Sócrates: Introducción a la práctica filosófica. Valencia: Diálogo: Editilde.

Canfux, V. (1996). Tendencias pedagógicas contemporáneas. Ibagué: Corporación Universitaria.

Cerletti, A. (2008). La enseñanza de la filosofía como problema filosófico. Buenos Aires: El Zorzal.

Espinel, Ó., \& Pulido, O. (2017). Enseñanza de la filosofía. Entre experiencia filosófica y ensayo. Universitas Philosophica, 34(69), 121-142. https://doi.org/10.11144/Javeriana.uph34-69.efee

Foucault, M. (1990). Tecnologías del yo. Barcelona: Paidós.

Foucault, M. (1991) “El sexo como moral”. En: Saber y verdad. Ediciones la Piqueta.

Foucault, M. (1994). Hermenéutica del sujeto. Madrid: La Piqueta, Madrid.

Foucault, M. (1999). El retorno de la moral. En Obras esenciales: Vol. III. Estética, ética y hermenéutica (pp. 382-390). Barcelona: Paidós.

Habermas (1989). Teoría de la acción comunicativa. Madrid: Taurus.

Hadot, P. (1998). ¿Qué es la filosofía antigua? México: FCE.

Hadot, P. (2006). Ejercicios espirituales y filosofía antigua. Madrid: Ediciones Siruela S.A.

Hadot, P. (2009). La Filosofía como forma de vida: Conversaciones con Jeannie Carlier y Arnold I. Davidson. Barcelona: Alpha Deca.

Kohan, W. (2009). Sócrates: El enigma de enseñar. Buenos Aires: Biblos.

Kant, I. (1943). Sobre el saber filosófico. Madrid: Madrid.

León, A. (2006). ¿Enseñar filosofía? Cali: Programa editorial. Universidad del valle. 
Londoño, R. (1985). El discurso reactivo de la enseñanza escolar de la filosofía: Colombia 1945-1970. Educación y ciencia 1(1), UPTC, Tunja.

Londoño R, (2002). Etica del goce y la educación en la neo-modernidad. Cuestiones de filosofía, (2-3), UPTC, Tunja. https://revistas.uptc. edu.co/index.php/cuestiones_filosofia

Luengo, J. (2004). La Educación como objeto de conocimiento: El concepto de Educación. En M. del M. del Del Pozo (Ed.), Teorías e instituciones contemporáneas de educación (pp. 45-60). Madrid: Biblioteca Nueva.

Mársico, C. (2017). La noción de Parrhesía en M. Foucault a la luz de las filosofías del círculsocrático. Revista de Filosofía, 4, (3) http://www. redalyc.org/articulo.oa?id=471557051003

Ministerio de Educación Nacional. (2010). Orientaciones pedagógicas para la filosofía en la educación media (Ministerio de Educación Nacional). Bogotá.

Nehamas, A. (2006). El arte de vivir: Reflexiones socráticas de Platón a Foucault. Valencia: Pre-Textos

Nussbaum, M. (2013). Sin fines de lucro. Por qué la democracia necesita de las humanidades. Buenos Aires: Katz.

Obiols, G. (2008). Una introducción a la enseñanza de la filosofía. Buenos Aires: Libros del Zorz 29. Recuperado de https://journals. epistemopolis.org/index.php/gestion/article/view/48

Perea, A. (2017). De la actitud crítica como vida filosófica: Verdad, poder $y$ espiritualidad en Foucault. http://www.redalyc.org/articulo. oa?id=105152132004

Piaget, J. (1987). Psicología y pedagogía. Barcelona: Ariel

Pulido, O. (2018). La universidad como proyecto modernizador. Ilusiones $y$ desencantos. Tunja: editorial UPTC.

Redacción El Heraldo.co. (2016, agosto 14). ¿De qué hablan hoy los filósofos colombianos? El Heraldo Recuperado 14 de abril de 2019, de https://www.elheraldo.co/tendencias/de-que-hablan-hoy-losfilosofos-colombianos-278245

Tozzi, M. (2007). Sobre la didáctica del aprendizaje del filosofar. Diálogo filosófico, 68, 207-215.

Unesco. (2011). La filosofía, una escuela de la libertad: Enseñanza de la filosofía y aprendizaje del filosofar : la situación actual y las perspectivas para el futuro. Paris: Unesco. 
Zubiría, J. (2018). La reforma pedagógica pendiente en la universidad. Recuperado 7 de julio de 2019, de La reforma pedagógica que propone Julián de Zubiría para salvar las universidades website: https:// www.semana.com/educacion/articulo/la-reforma-pedagogica-quepropone-julian-de-zubiria-para-salvar-las-universidades/587724 